\title{
Testing the Cue Dependence of Problem-Solving-Induced Forgetting
}

\author{
Benjamin C. Storm and Rebecca H. Koppe/ ${ }^{1}$
}

\begin{abstract}
:
Thinking and remembering can cause forgetting. In the context of remembering, retrieving one item can cause the forgetting of other items (Anderson, Bjork, \& Bjork, 1994). A similar phenomenon has been observed in the context of creative problem solving - attempting to generate a target associate in the Remote Associates Test (RAT) can cause the forgetting of inappropriate associates (Storm, Angello, \& Bjork, 2011). Experiment 1 examined whether this problem-solving-induced forgetting is cue dependent or cue independent by manipulating the cues used at final test. Whereas some participants were tested on the inappropriate associates using the same cues that were used during problem solving, other participants were tested using new, or independent, cues. Problem-solving-induced forgetting was observed in the same-cue condition, but not in the new-cue condition. Experiment 2 replicated the overall absence of problem-solvinginduced forgetting in the new-cue condition and found that individual differences in cue-independent forgetting did not predict problem-solving performance on a separate set of RAT problems.
\end{abstract}

\section{Keywords:}

problem solving, memory, forgetting, retrieval-induced forgetting, cue independence, fixation

\footnotetext{
${ }^{1}$ University of Illinois at Chicago
} 


\section{Introduction}

Solving problems can be difficult, especially when useful information is less accessible than nonuseful information. In the Remote Associate Test (RAT; Mednick, 1962), for example, generating an appropriate solution can be difficult because inappropriate solutions get in the way. Participants are presented three cue words (e.g., bass-complex-sleep) and asked to generate a target associate that is related to each of the cue words. The target associate (i.e., deep) can be a synonym, share a general semantic relationship, or combine with the cue words to form a commonly spoken phrase. RAT problems are particularly difficult because the strongest associates for each cue word often bear no relationship to the other cue words. For example, although the cue sleep may be highly associated with rest and dream, neither of these words is associated with bass or complex and would not, therefore, serve as appropriate solutions. To solve a RAT problem, one must bypass inappropriate associates in order to access a more remote target associate.

Smith and Blankenship (1991) found that performance on the RAT can be further impaired by exposing participants to inappropriate associates prior to problem solving. For example, participants exposed to cat-nap, sleep-night, and board-wood were significantly less likely to generate a solution to the problem cat-sleep-board than participants who were not given such exposure. In this example, nap, night, and wood are likely to block the participant's ability to generate the target associate, walk. Similarly, Wiley (1998) found that when RAT problems are designed to automatically activate irrelevant associations within a domain, participants with more domain knowledge solved fewer problems than participants with less domain knowledge. Specifically, baseball experts solved fewer RAT problems than baseball novices when baseball knowledge was activated by a given problem but did not lead to a viable solution. These findings demonstrate how inappropriate associates can cause mental fixation and impair the successful generation of new and appropriate associates (for a review, see Smith, 2003).

Ironically, one way people are able to overcome fixation is by taking a break from problem solving. Recent work by Collier and Beeman (2012), for example, has shown that unsolved RAT problems can be solved after a delay and especially when problem solvers have an intuitive sense that inaccessible solutions are in mind. According to the forgetting-fixation hypothesis, stopping one's attempt to generate a solution may provide the time or contextual change necessary for the misleading and irrelevant associates to be forgotten (e.g., Smith, 1995; Smith \& Blankenship, 1989; 1991; Smith, Sifonis, \& Angello, 2012). Although misleading associates may interfere with a participant's ability to retrieve a solution initially, an incubation period may reduce access to those interfering items and thus facilitate access to the hidden solution. Storm and colleagues have suggested that problem solvers may also have the capacity to overcome fixation during problem solving by actively inhibiting, and thus forgetting, fixation-inducing associates (Storm \& Angello, 2010; Storm, Angello, \& Bjork, 2011; for a review see Storm, 2011a). One finding that sup-

- volume 4, no. 2 (Spring 2012) 
ports this hypothesis is the correlation between RAT problem solving and retrieval-induced forgetting (Storm \& Angello, 2010).

Retrieval-induced forgetting is observed when the retrieval of a subset of items in memory causes the forgetting of other items in memory (Anderson, Bjork, \& Bjork, 1994). In a typical retrieval-induced forgetting paradigm, participants are exposed to a list of category-exemplar pairs (fruit-lemon, weapon-sword, fruit-banana, weapon-bomb) and then receive retrieval practice for half of the exemplars from half of the categories (e.g., fruit-le___. Retrieval-induced forgetting is observed when unpracticed items from practiced categories (i.e., banana) are recalled less well on a final cued-recall test than are unpracticed items from unpracticed categories (i.e., sword, bomb). The phenomenon has been shown to be highly robust and general, occurring in many contexts and with a variety of materials (for reviews, see, Anderson, 2003; Levy \& Anderson, 2002). According to the inhibitory account of retrieval-induced forgetting, the retrieval-practice cues activate nontarget items as well as target items. Inhibition is elicited to resolve interference by suppressing the accessibility of the nontarget items in order to facilitate the retrieval of the target items, thus causing the nontarget items to be forgotten (Anderson, 2003; Storm, 2011b).

Storm and Angello (2010) argued that the inhibitory process underlying retrievalinduced forgetting might also underlie the ability to overcome fixation during creative problem solving. To test this hypothesis they examined the correlation between retrievalinduced forgetting and RAT problem solving. If inhibition does provide a mechanism by which to overcome fixation, then individuals who exhibit more retrieval-induced forgetting should exhibit a greater ability to overcome fixation in the RAT.

In the first phase of their experiment, participants attempted to solve 20 RAT problems; half of the participants were exposed to inappropriate associates prior to problem solving (fixation condition), half were not (baseline condition). Participants in the fixation condition were exposed to cat-nap, sleep-night, and board-wood, for example, before attempting to generate a common associate to cat-sleep-board. Retrieval-induced forgetting was then measured in the second phase of the experiment using a close variant of the paradigm described above (Anderson et al., 1994). As expected, participants exposed to fixating associates solved significantly fewer RAT problems than participants in the baseline condition. More importantly, the extent to which participants suffered fixation was moderated by individual differences in retrieval-induced forgetting. Participants who exhibited the least retrieval-induced forgetting were most impaired by exposure to fixating associates, whereas participants who exhibited the most retrieval-induced forgetting were least impaired.

Admittedly, Storm and Angello's (2010) evidence for the role of inhibition and forgetting in problem solving is indirect. If inhibition does act to reduce the accessibility of fixating associates then one should be able to directly observe the consequences of such 
inhibition. In subsequent work, Storm et al. (2011) examined this possibility by having participants study a series of cue-response pairs prior to RAT problem solving. This time, however, only half of the cues from the word pairs formed the to-be-solved RAT problems. Consequently, half of the response words served as fixating responses and half did not. After attempting to solve the problems, participants were given a surprise cued-recall test for the response words. In each of three experiments, fixating responses were recalled significantly less often than nonfixating responses, thus demonstrating problem-solvinginduced forgetting.

Problem-solving-induced forgetting appears to share certain properties with retrieval-induced forgetting. For example, just as retrieval success is not a necessary condition for retrieval-induced forgetting (Storm, Bjork, Bjork, \& Nestojko, 2006; Storm \& Nestojko, 2010), problem-solving success is not a necessary condition for problem-solving-induced forgetting (Storm et al., 2011). Attempting to solve problems caused participants to forget fixating associates even when those attempts failed. In fact, problem-solving-induced forgetting was observed when problem solving was made impossible, thus ensuring that participants would be unable to generate any viable response. This finding makes sense from an inhibitory perspective as it is not solving the problem per se that causes forgetting. Rather, it is the inhibition elicited to resolve competition from fixating associates that causes forgetting and such inhibition should occur regardless of whether a problem is eventually solved.

It is important to note that although problem-solving-induced forgetting is not contingent on problem-solving success, it may facilitate problem-solving success when problem solving is possible. For instance, in their third experiment, Storm et al. (2011) examined the correlation between problem-solving-induced forgetting and problemsolving performance on a separate set of fixated RAT problems. Just as retrieval-induced forgetting correlated positively with problem-solving performance, so did problemsolving-induced forgetting. Specifically, participants who exhibited more forgetting as a consequence of problem solving were more likely to solve a separate set of RAT problems (under fixated conditions) than participants who exhibited less forgetting, a finding which provides additional evidence that problem-solving-induced forgetting plays an adaptive role in problem solving.

\section{Cue-Dependent vs. Cue-Independent Forgetting}

Many questions remain concerning the nature and dynamics of problem-solvinginduced forgetting. One question we sought to address in the current paper is whether the forgetting is cue dependent or cue independent. Some theorists have argued that inhibitory-based forgetting should be observed when a response is tested given a new or independent retrieval cue (e.g., Anderson, 2003; Anderson \& Spellman, 1995; but see Perfect, Stark, Tree, Moulin, Ahmed, \& Hutter, 2004). For example, the item banana should 
not only become less recallable when tested using the category cue fruit, but also when tested using a new cue, such as monkey. Many studies have tested this prediction in the context of retrieval-induced forgetting and a good number of them have provided evidence of cue-independent forgetting (e.g., Anderson \& Bell, 2001; Anderson, Green, \& McCulloch, 2000; Anderson \& Spellman, 1995; Johnson \& Anderson, 2004; MacLeod \& Saunders, 2005; Radvansky, 1999; Saunders \& MacLeod, 2006), whereas others have not (e.g., Camp, Pecher, \& Schmidt, 2005; 2007; Perfect et al., 2004; Williams \& Zacks, 2001).

One reason researchers have been so interested in cue independence is that it has been argued to help distinguish between inhibitory and noninhibitory forms of forgetting. Although there are many findings that support the inhibitory account and challenge noninhibitory accounts (for reviews, see Anderson, 2003; Bäuml, Pastötter, \& Hanslmayr, 2010; Storm, 2011b), cue independence is regarded by some to be one of the most important findings. According to noninhibitory accounts, retrieval-induced forgetting occurs because a subset of items related to the retrieval-practice cues are strengthened, which then occludes, interferes with, or steals activation away from other items associated with those cues (see e.g., Anderson, 1983; McGeoch, 1942; Raaijmakers \& Shiffrin, 1981; Rundus, 1973). None of these noninhibitory accounts can account for retrieval-induced forgetting being cue independent (Anderson \& Spellman, 1995).

Somewhat less clear-at least to the current authors-is why inhibitory-based forgetting needs to be cue independent. Anderson and colleagues have argued that cue-independent forgetting occurs because inhibition acts at the level of an item's representation to decrease the activation of that item directly, thus rendering it less accessible irrespective of the cue provided at test. However, it is unclear why inhibition needs to act at the level of an item's representation. At the crux of the inhibitory account is the idea that competing nontarget items are suppressed in order to facilitate the retrieval of a target item-and this process could be accomplished by suppressing the accessibility of nontarget items given all potential cues or by suppressing the accessibility of nontarget items given the particular cues that were available during retrieval practice. Either way, the forgetting observed could still be considered the consequence of inhibition.

In fact, if one assumes that inhibition acts to resolve future interference-in addition to current interference-cue-dependent forgetting might be considered to be more adaptive than cue-independent forgetting. That is, to adaptively update the future accessibility of items in memory, inhibition might act to make nontarget items less accessible in response to only the cue or cues that prompted their inappropriate activation. An important benefit of cue-dependent forgetting is that interference can be resolved without rendering inhibited items inaccessible given all potential cues. Often, information that we do not want in one context is still very important in other contexts, and it would be useful for that information to retain its accessibility in those contexts (Bjork \& Bjork, 1992). To accomplish this form of cue-specific forgetting, inhibition might act on 
the association between a cue and response to render that response inaccessible given that specific cue-but not given other, potentially useful, cues.

In the context of problem-solving-induced forgetting, fixating associates may be inhibited given the problem-solving cues from which they were activated, but not given other, independent retrieval cues. We tested this prediction in the current study by employing a paradigm very similar to that of Storm et al. (2011). Participants first studied a list of cue-response pairs and then attempted to solve a series of RAT problems. The consequences of this problem solving were then observed on a surprise final test. Half of the participants were shown the same cue words and asked to recall the earlier-learned response words. The other half were shown new cue words and asked to recall the earlierlearned response words. If problem-solving-induced forgetting is cue independent then forgetting should be observed on both versions of the final test. If problem-solving-induced forgetting is cue dependent, however, then we should only observe forgetting when the same cues are provided.

\section{Experiment 1}

\section{Method}

\section{Participants}

A total of 86 undergraduates ( 15 male; 71 female) from the University of Illinois at Chicago (UIC) participated for course credit in an introductory psychology course ( $M$ years of age $=19.2$ ). All participants provided informed consent.

\section{Materials}

Fifty-four word pairs were selected (e.g., silk-worm, ruin-mess, secret-quiet), each consisting of a cue-response pair of moderate forward-associative strength $(M=.10, S D=.03$; Nelson, McEvoy, \& Schrieber, 1998). The cue words from each of the pairs were then grouped into sets of three to form 18 impossible RAT problems (e.g., silk-ruin-secret). We employed impossible RAT problems because Storm et al. (2011) observed the largest problem-solvinginduced forgetting effects using such problems. For counterbalancing purposes, the 54 word pairs (and 18 associated RAT problems) were divided into two subsets. Although participants studied all 54 pairs, any given participant would only attempt to solve 9 of the 18 RAT problems. Responses associated with to-be-solved RAT problems will be referred to as fixating responses; the other responses will be referred to as baseline responses. Finally, 5 possible RAT problems were interleaved with the impossible RAT problems to keep participants from becoming suspicious about the problems. The 15 cue words used to create the possible RAT problems were also paired with response words of moderate forward associative strength.

- volume 4, no. 2 (Spring 2012) 


\section{Procedure}

The experiment consisted of three main phases: cue-response training, RAT problem solving, and a cue-response final test.

\section{Cue-response training}

Participants were first given a single sheet of paper with 69 cue-response pairs presented in three columns and in a random interleaved order. Participants were told to study the pairs for a total of $4 \mathrm{~min}$ and that in a later part of the experiment they would be asked to recall the response words given the cue words. Immediately following study, participants were given another sheet of paper with the same 69 cue-response pairs listed in the same order. This time, only the first two letters of each response word were provided and participants were given $3 \mathrm{~min}$ to write the responses. Cue-response training was implemented to ensure that the cue-response pairs were sufficiently strengthened to cause fixation during problem solving.

\section{RAT problem solving}

Participants were given 8 min to solve 14 RAT problems listed on a single sheet of paper. Problems 1, 4, 7, 10, and 13 were always possible; the rest were impossible. The particular set of impossible problems that participants attempted to solve was counterbalanced across participants. Half of the participants attempted to solve the 9 RAT problems associated with one set of 27 cue-response pairs; the other half attempted to solve the 9 RAT problems associated with the other set of 27 cue-response pairs. All participants were given instructions and a practice example before attempting to solve the problems. They were told to try to think of a fourth word that was either semantically related, a synonym, or formed a commonly spoken phrase with each of the three cue words. Finally, participants were warned that the problems were created by combining the cue words that had been previously learned and it was emphasized that none of the solutions would ever be one of the associated response words.

\section{Cue-response final test}

Participants were randomly assigned to one of two between-subject final-test conditions (same cue vs. new cue). Participants in the same-cue condition $(n=44)$ received the same final test employed in Storm et al.'s (2011) Experiment 3. Specifically, all 69 cues were listed on a single sheet of paper and participants were given $4 \mathrm{~min}$ to recall the associated responses. The cues were listed in alphabetical order (a different order from which they were studied) and the instructions clearly stated that participants were to recall the response words that had been learned during the first part of the experiment. Participants in the new-cue condition $(n=42)$ received a similar test except the cues were replaced by new cues. For example, rather than provide the cue silk for the response worm, participants were provided the cue slug: wo . The first two letters of the response word were provided to ensure that performance was comparable in the two conditions (the use of 
letter stems at final test in studies of retrieval-induced forgetting is very common). The new cues were chosen to have approximately the same forward associative strength as the cues they replaced ( $M=.09, S D=.05$; Nelson et al., 1998). As in the same-cue condition, the new cues were listed in alphabetical order and participants were explicitly told that new cues were being provided and that they were to recall the response words that had been learned during the first part of the experiment.

\section{Results}

Performance on the cue-response final test is shown in the top half of Table 1 as a function of item type and test format. The data were analyzed using a 2 (fixating responses vs. baseline responses) $\times 2$ (same cue vs. new cue) mixed-design Analysis of Variance (ANOVA) with item type serving as the only repeated measure. As expected, a main effect of problem-solving-induced forgetting was observed such that fixating responses $(M=78.4 \%, S E=2.0 \%)$ were recalled significantly less well than baseline responses $(M=$ $80.8 \%, S E=1.9 \%), F(1,84)=6.66, M S E=.004, p=.01, \eta_{p}{ }^{2}=.07$. However, this main effect was qualified by a significant interaction, $F(1,84)=4.75, M S E=.004, \mathrm{p}=.03, \eta_{p}^{2}=.05$. Participants in the same-cue condition exhibited significant problem-solving-induced forgetting, $t(43)=3.27, p=.002, d=.50$, whereas participants in the new-cue condition did not, $t(41)=0.29, p=.77, d=.05$.

\section{Experiment 2}

The results of the first experiment suggest that problem-solving-induced forgetting is cue dependent-fixating responses only became inaccessible in relation to the specific cues provided during problem solving. We sought to replicate this finding in the second experiment while also measuring each participant's problem-solving performance on a separate set of problems. Storm et al. (2011) found that the extent to which participants exhibited problem-solving-induced forgetting predicted their performance on a separate set of RAT problems. Specifically, participants who exhibited more forgetting solved more problems. This correlation is important for a number of reasons, but especially because it is consistent with the idea that problem-solving-induced forgetting is the consequence of a mechanism that facilitates problem solving by attenuating fixation. If the forgetting was caused by a different mechanism, such as blocking or associative interference, then individuals who exhibited more forgetting should have been, if anything, more susceptible to the interfering effects of fixating response words.

In Experiment 2 we examined whether cue-independent problem-solving-induced forgetting predicts problem-solving performance in the same way that Storm et al. (2011) found cue-dependent problem-solving-induced forgetting to predict problem-solving performance. Specifically, will participants exhibiting greater levels of cue-independent 
problem-solving-induced forgetting be better at solving a separate set of RAT problems than participants exhibiting less such forgetting? Even if we again fail to observe belowbaseline cue-independent forgetting, there is still going to be variability among subjects, and if individuals who do exhibit cue-independent problem-solving-induced forgetting do so as a consequence of inhibition, then such individuals should exhibit superior performance on a separate set of fixated RAT problems.

\section{Method}

\section{Participants}

Forty-six UIC undergraduates (10 male; 36 female) participated for course credit in an introductory psychology course ( $M$ years of age $=20.5$ ). All participants provided informed consent.

\section{Measuring problem-solving performance}

Problem-solving performance was measured in the same way as in Storm et al.'s (2011) Experiment 3. Participants were first given cue-response training for 60 fixation-inducing pairs and then $18 \mathrm{~min}$ to solve 20 possible RAT problems. The materials were taken directly from Storm and Angello (2010), who selected their RAT problems from Mednick and Mednick (1967) and Bowers, Regehr, Balthazard, and Parker (1990). The cue-response pairs were presented simultaneously on a single sheet of paper and each consisted of a cue word that would be used in one of the 20 RAT problems. Participants were told that they would be tested on their ability to recall the response words given the cue words. After 4 min of study, participants were given 3 min to retrieve the response words given cue-plus-one-letter-stem retrieval cues. This retrieval practice served to strengthen the cue-response associations, thereby increasing the likelihood of experiencing fixation during subsequent RAT problems. RAT problem solving immediately followed cue-response training. All 20 RAT problems appeared on a single sheet of paper and participants were warned that the earlier learned response words would never be viable solutions. Corrective individualized feedback was provided every $6 \mathrm{~min}$ to ensure that participants continued to attempt to solve incorrectly answered problems. Performance was measured as the total number of problems solved after $18 \mathrm{~min}$ of problem solving.

\section{Measuring problem-solving-induced forgetting}

The materials and procedure were identical to those employed in the new-cue condition of Experiment 1. None of the RAT problems or cue-response pairs were repeated from the earlier problem-solving performance phase of the experiment.

\section{Results}

In the first phase of the experiment, which was designed to measure problem-solving performance under fixation, participants solved $35.5 \%$ (SE $=2.7 \%$ ) of the problems. In the 
second phase of the experiment participants were tested on their ability to recall fixating and baseline response words following RAT problem solving. As can be seen in Table 1, we once again failed to find any evidence of cue-independent problem-solving-induced forgetting. In fact, we found a significant effect of problem-solving-induced facilitation such that fixating responses were recalled more effectively than baseline responses, $t(45)$ $=2.12, p=.04, d=.32$. When we examined the relationship between problem-solvinginduced forgetting and problem-solving performance, a significant correlation failed to emerge $(r=-.09, p=.55)$. These data provide further evidence that problem-solvinginduced forgetting is cue dependent and, moreover, that variance in such forgetting is not indicative of problem-solving ability. It is unclear why we observed facilitation in this experiment, whereas we did not in the first experiment. The only difference between the two experiments was that participants in Experiment 2 attempted to solve the 20 possible RAT problems before beginning the phase designed to measure problem-solvinginduced forgetting.

We next compared the current results with those observed by Storm et al. (2011; Exp. 3), which are also shown in Table 1. We felt it was appropriate to make this direct comparison because (1) with the exception of the nature of the final test, the exact same materials and procedures were used, and, (2) participants were randomly assigned from the same sample to the two experiments. First, to examine problem-solving-induced forgetting we employed a 2 (fixating responses vs. baseline responses) $\times 2$ (same cue vs. new cue) mixed-design ANOVA, with participants in the same-cue condition coming from Storm et al. (2011; Exp. 3) and participants in the new-cue condition coming from the current experiment. A significant interaction was found, $F(1,86)=13.96, M S E=.006$, $p<.001$, partial $\eta^{2}=.14$.

To test whether the correlation between problem-solving-induced forgetting and problem-solving performance was moderated by the nature of the final test, we ran a regression analysis examining the proportion of variance in RAT problem-solving performance that could be explained by type of test, problem-solving-induced forgetting, and the type of test $X$ problem-solving-induced forgetting interaction. The main effects were entered first to assess whether the interaction term could account for significant additional variance. Although the complete model did not reach significance, $F(3,84)=$ $1.99, p=.12, R^{2}=.07$, the type of test $\mathrm{X}$ problem-solving-induced forgetting interaction term accounted for significant additional variance, $F(1,84)=4.30, p=.04, \Delta R^{2}=.05$. This finding confirms that the relationship between problem-solving-induced forgetting and problem-solving performance was moderated by the nature of the test used to measure problem-solving-induced forgetting. Specifically, problem-solving-induced forgetting observed on the same-cue final test was significantly more positively correlated with problem-solving performance $(r=+.36)$ than was problem-solving-induced forgetting observed on the new-cue final test $(r=-.09)$.

- volume 4, no. 2 (Spring 2012) 
Table 1

Mean Percentage of Response Words Correctly Recalled during the Final Cue-Response Tests of Experiments 1 and 2.

\begin{tabular}{|c|c|c|c|c|c|c|}
\hline \multirow{3}{*}{$\begin{array}{l}\text { Experiment } \\
\text { Test Format }\end{array}$} & \multicolumn{6}{|c|}{ Item Type } \\
\hline & \multicolumn{2}{|c|}{ Baseline Responses } & \multicolumn{2}{|c|}{ Fixating Responses } & \multicolumn{2}{|c|}{ Forgetting Effect } \\
\hline & $M$ & $S E$ & $M$ & $S E$ & $M$ & $d$ \\
\hline \multicolumn{7}{|l|}{ Experiment 1} \\
\hline Same Cue & $85.1 \%$ & $2.5 \%$ & $80.6 \%$ & $2.7 \%$ & $4.5 \%$ & .50 \\
\hline New Cue & $76.6 \%$ & $2.9 \%$ & $76.2 \%$ & $3.0 \%$ & $0.4 \%$ & .05 \\
\hline \multicolumn{7}{|l|}{ Experiment 2} \\
\hline Same Cue* & $77.4 \%$ & $2.5 \%$ & $72.0 \%$ & $2.7 \%$ & $5.4 \%$ & .49 \\
\hline New Cue & $62.3 \%$ & 2.4 & $66.0 \%$ & $2.6 \%$ & $-3.7 \%$ & -.32 \\
\hline
\end{tabular}

*Same-cue data from Storm, Angello, and Bjork (2011; Exp. 3) is shown under Experiment 2 for purposes of comparison. SE shows the Standard Error of the Mean; $d$ shows the Cohen's Effect Size of the Difference between Fixation and Baseline (problem-solving-induced forgetting).

\section{General Discussion}

Mental fixation can impair creative thinking and problem-solving performance (Smith \& Blankenship, 1989; 1991; for a review, see Smith, 2003). Storm et al. (2011) argued that one way in which people are able to overcome fixation is by forgetting the associates that cause fixation via problem-solving-induced forgetting. The current study found that whether problem-solving-induced forgetting is observed depends on the particular cues provided at final test. When the same cues were provided (i.e., the cues used during cue-response training and RAT problem solving), significant problem-solving-induced forgetting was observed. When new cues were provided (i.e., cues that were independent of cue-response training and RAT problem solving), problem-solving-induced forgetting was not observed. This finding suggests that problem-solving-induced forgetting is cue dependent.

Not only did we replicate the absence of cue-independent problem-solving-induced forgetting in Experiment 2, we actually observed significant problem-solving-induced facilitation. That is, participants became more likely to recall fixating responses than baseline responses. Although an intriguing observation, we think it is best to treat this finding with caution because facilitation was not observed in Experiment 1 and because it is unclear why the results should have differed between the two experiments. If the facilitation effect is reliable, however, it might suggest that depending on the nature of the final test problem solving can both enhance and impair memory of fixating associates. More research will be necessary to explore when and why facilitation might occur. 
Another interesting finding was that individual differences in cue-independent problem-solving-induced forgetting failed to predict performance on a separate set of fixated RAT problems. In contrast to Storm et al. (2011), who found a significant positive correlation between problem-solving performance and problem-solving-induced forgetting, we found a nonsignificant negative correlation. The only difference between the two experiments was the nature of the final test. Whereas problem-solving-induced forgetting observed on a same-cue final test does predict problem-solving performance, problem-solving-induced forgetting observed on a new-cue final test does not. This difference suggests that the source of the variability in the recall of fixating responses on the new-cue final test is unrelated to one's ability to solve fixated RAT problems. Participants who became more likely to recall fixating responses were just as capable of solving fixated RAT problems as were participants who became less likely to recall fixating responses.

One potential benefit of problem-solving-induced forgetting being cue dependent is that fixating responses can be inhibited, thus resolving fixation, but without rendering fixating responses inaccessible in other potentially useful contexts. For example, although certain fixating responses may be inappropriate for solving one problem, those same responses may be very appropriate for solving another problem. Thus, even if an associate is forgotten, the cue-dependent nature of forgetting may ensure that it retains its accessibility should it become relevant for solving a future problem.

An important question, however, is why problem-solving-induced forgetting is cue dependent if retrieval-induced forgetting is cue independent. If the two phenomena are caused by a similar inhibitory process then it seems likely that the consequences of that process should demonstrate similar properties. One possibility is that retrievalinduced forgetting is not cue independent. Although there is considerable evidence that retrieval-induced forgetting is cue independent, some theorists remain skeptical (for various opposing views, see e.g., Camp, Pecher, Schmidt, \& Zeelenberg, 2009; Perfect et al., 2004), and the current work may provide evidence against it. Another possibility is that inhibition acts somewhat differently in the context of problem-solving-induced forgetting than it does in the context of retrieval-induced forgetting. Perhaps, for example, retrieval-induced forgetting is more likely reflect the inhibition of an item's representation, whereas problem-solving-induced forgetting is more likely to reflect the inhibition of the cue-response association.

A third possibility is that problem-solving-induced forgetting is not caused by inhibition at all. If cue independence is a necessary criterion for inhibition, which some theorists have argued, then the current results suggest that problem-solving-induced forgetting cannot be considered to be a consequence of inhibition. However, from our perspective, we do not believe cue independence is a necessary criterion for inhibition. Although cue independence provides powerful evidence against noninhibitory accounts of forgetting,

- volume 4, no. 2 (Spring 2012) 
it is unclear why it should be a necessary property of inhibitory-based forgetting. After all, a process that acts to resolve competition by reducing the accessibility of inappropriate responses in response to a specific cue can still be considered an inhibitory process even if the consequent forgetting is cue dependent. Storm et al.'s (2011) observation that individuals who exhibit greater levels of cue-dependent problem-solving-induced forgetting solve significantly more RAT problems than individuals who exhibit less forgetting is certainly consistent with this conjecture.

Of course, if problem-solving-induced forgetting is cue dependent then it will be necessary for inhibitory proponents to provide other forms of evidence supporting the inhibitory account. For example, there is evidence that retrieval-induced forgetting is competition dependent - that the extent to which items suffer retrieval-induced forgetting is determined by the extent to which they compete during retrieval practice (e.g., Anderson et al., 1994; Storm, Bjork, \& Bjork, 2007; for a review, see Storm, 2011b). There is also evidence that retrieval-induced forgetting is strength independent-that the extent to which practiced items are strengthened does not determine whether unpracticed items are forgotten (e.g., Anderson, Bjork, \& Bjork, 2000; Bäuml, 2002; Storm et al., 2006). Research examining individual differences has also supported the inhibitory account. For example, as long as noninhibitory sources of forgetting are controlled, individuals with inhibitory deficits tend to show reduced levels of retrieval-induced forgetting compared to individuals without such deficits (e.g., Soriano, Jiménez, Román, \& Bajo, 2009; Storm \& White, 2010). Finally, recent neuroimaging work has also provided compelling support for the inhibitory account (e.g., Kuhl, Dudukovic, Kahn, \& Wagner, 2007; Wimber, Rutschmann, Greenlee, \& Bäuml, 2009). Future work should adopt these lines of research to test the inhibitory account of problem-solving-induced forgetting.

In summary, the current findings provide important insight into the relationship between problem solving and memory. Attempting to solve a RAT problem can cause the forgetting of fixating associates, but such forgetting is specific to instances in which the cues provided during problem solving are also provided at test. Although this finding is consistent with noninhibitory accounts of problem-solving-induced forgetting (such as blocking and strength-based interference), we do not believe it necessarily refutes the inhibitory account. In fact, cue-dependent forgetting may provide an adaptive advantage for problem solvers by allowing them to inhibit fixating associates in one context while retaining access to those associates in other contexts. If this is the case, then future researchers will need to employ other methods to test the inhibitory account of problemsolving-induced forgetting. 


\section{References}

Anderson, J. R. (1983). The Architecture of Cognition. Cambridge, MA: Harvard University Press.

Anderson, M. C. (2003). Rethinking interference theory: Executive control and the mechanisms of forgetting. Journal of Memory and Language, 49, 415-445.

Anderson, M. C., \& Bell, T. (2001). Forgetting our facts: The role of inhibitory processes in the loss of propositional knowledge. Journal of Experimental Psychology: General, $130,544-570$.

Anderson, M. C., \& Spellman, B. A. (1995). On the status of inhibitory mechanisms in cognition: Memory retrieval as a model case. Psychological Review, 102, 68-100.

Anderson, M. C., Bjork, E. L., \& Bjork, R. A. (1994). Remembering can cause forgetting: Retrieval dynamics in long-term memory. Journal of Experimental Psychology: Learning, Memory, and Cognition, 20, 1063-1087.

Anderson, M. C., Bjork, E. L., \& Bjork, R. A. (2000). Retrieval-induced forgetting: Evidence for a recall-specific mechanism. Psychonomic Bulletin \& Review, 7, 522-530.

Anderson, M. C., Green, C., \& McCulloch, K. C. (2000). Similarity and inhibition in long-term memory: Evidence for a two-factor theory. Journal of Experimental Psychology: Learning, Memory, and Cognition, 26, 1141-1159.

Bäuml, K. -H. (2002). Semantic generation can cause episodic forgetting. Psychological Science, 13, 356-360.

Bäuml, K. -H., Pastötter, B., \& Hanslmayr, S. (2010). Binding and inhibition in episodic memory - Cognitive, emotional, and neural processes. Neuroscience \& Biobehavioral Reviews, 34, 1047-1054.

Bjork, R. A., \& Bjork, E. L. (1992). A new theory of disuse and an old theory of stimulus fluctuation. In A. Healy, S. Kosslyn, \& R. Shiffrin (Eds.), From learning processes to cognitive processes: Essays in honor of William K. Estes (Vol. 2, pp. 35-67). Hillsdale, NJ: Lawrence Erlbaum Associates, Inc.

Bowers, K. S., Regehr, G., Balthazard, C. G., \& Parker, K. (1990). Intuition in the context of discovery. Cognitive Psychology, 22, 72-110.

Camp, G., Pecher, D., \& Schmidt, H. G. (2005). Retrieval-induced forgetting in implicit memory tests: The role of test awareness. Psychonomic Bulletin \& Review, 12, 490-494.

Camp, G., Pecher, D., \& Schmidt, H. G. (2007). No retrieval-induced forgetting using itemspecific independent cues: Evidence against a general inhibitory account. Journal of Experimental Psychology: Learning, Memory, and Cognition, 33, 950-958.

Camp, G., Pecher, D., Schmidt, H. G., \& Zeelenberg, R. (2009). Are independent probes truly independent? Journal of Experimental Psychology: Learning, Memory, and Cognition, 35, 934-942.

Collier, A. K., \& Beeman, M. (2012). Intuitive tip of the tongue judgments predict subsequent problem solving one day later. Journal of Problem Solving, 4(2), Article 8.

- volume 4, no. 2 (Spring 2012) 
Johnson, S. K., \& Anderson, M.C. (2004). The role of inhibitory control in forgetting semantic knowledge. Psychological Science, 15, 448-453.

Kuhl, B. A., Dudukovic, N. M., Kahn, I., \& Wagner, A. (2007). Decreased demands on cognitive control reveal the neural processing benefits of forgetting. Nature Neuroscience, 10, 908-914.

Levy, B. J., \& Anderson, M. C. (2002). Inhibitory processes and the control of memory retrieval. Trends in Cognitive Science, 6, 299-305.

MacLeod, M. D., \& Saunders, J. (2005). The role of inhibitory control in the production of misinformation effects. Journal of Experimental Psychology: Learning, Memory, and Cognition, 31, 964-979.

McGeoch, J. A. (1942). The psychology of human learning. New York: Longmans, Green.

Mednick, S. (1962). The associative basis of the creative problem solving process. Psychological Review, 69, 200-232.

Mednick, S. A., \& Mednick, M.T. (1967). Examiner's manual: Remote Associates Test. Boston, MA: Houghton Mifflin.

Nelson, D. L., McEvoy, C. L., \& Schreiber, T. A. (1998). The University of South Florida word association, rhyme, and word fragment norms. http://www.usf.edu/FreeAssociation/.

Perfect, T. J., Stark, L. J., Tree, J. J., Moulin, C. J. A., Ahmed, L., \& Hutter, R. (2004). Transfer appropriate forgetting: The cue-dependent nature of retrieval-induced forgetting. Journal of Memory and Language, 51, 399-417.

Raaijmakers, J. G., \& Shiffrin, R. M. (1981). Search of associative memory. Psychological Review, 88, 93-134.

Radvansky, G. A. (1999). Memory retrieval and suppression: The inhibition of situation models. Journal of Experimental Psychology: General, 128, 563-579.

Rundus, D. (1973). Negative effects of using list items as retrieval cues. Journal of Verbal Learning and Verbal Behavior, 12, 43-50.

Saunders, J., \& MacLeod, M. D. (2006). Can inhibition resolve retrieval competition through the control of spreading activation? Memory \& Cognition, 34, 307-322.

Smith, S. M. (1995). Fixation, incubation, and insight in memory and creative thinking. In S. M. Smith, T. B. Ward, \& R. A. Finke (Eds.), The creative cognition approach (pp. 135146). Cambridge, MA: MIT Press.

Smith, S. M. (2003). The constraining effects of initial ideas. In P. Paulus \& B. Nijstad (Eds.), Group Creativity: Innovation Through Collaboration (pp.15-31). New York, NY: Oxford University Press.

Smith, S. M., \& Blankenship, S. E. (1989). Incubation effects. Bulletin of the Psychonomic Society, 27, 311-314.

Smith, S. M., \& Blankenship, S. E. (1991). Incubation and the persistence of fixation in problem solving. American Journal of Psychology, 104, 61-87. 
Smith, S. M., Sifnois, C. M., \& Angello, G. (2012). Clue insensitivity in remote associates test problem solving. The Journal of Problem Solving, 4(2), Article 2.

Soriano, M. F., Jiménez, J. F., Román P., \& Bajo, M. T. (2009). Inhibitory processes in memory are impaired in schizophrenia: Evidence from retrieval-induced forgetting. British Journal of Psychology, 100, 661-673.

Storm, B. C. (2011a). The benefit of forgetting in thinking and remembering. Current Directions in Psychological Science, 20, 291-295.

Storm, B. C. (2011b). Retrieval-induced forgetting and the resolution of competition. In A. S. Benjamin (Ed.), Successful remembering and successful forgetting: A Festschrift in honor of Robert A. Bjork (pp. 89-105). New York, NY: Psychology Press.

Storm, B. C., \& Angello, G. (2010). Overcoming fixation: Creative problem solving and retrieval-induced forgetting. Psychological Science, 21, 1263-1265.

Storm, B. C., \& Nestojko, J. F. (2010). Successful inhibition, unsuccessful retrieval: Manipulating time and success during retrieval practice. Memory, 18, 99-114.

Storm, B. C., \& White, H. A. (2010). ADHD and retrieval-induced forgetting: Evidence for a deficit in the inhibitory control of memory. Memory, 18, 99-114.

Storm, B. C., Angello, G., \& Bjork, E. L. (2011). Thinking can cause forgetting: Memory dynamics in creative problem solving. Journal of Experimental Psychology: Learning, Memory, and Cognition, 37, 1287-1293.

Storm, B. C., Bjork, E. L., \& Bjork, R. A. (2007). When intended remembering leads to unintended forgetting. Quarterly Journal of Experimental Psychology, 60, 909-915.

Storm, B. C., Bjork, E. L., Bjork, R. A., \& Nestojko, J. F. (2006). Is retrieval success a necessary condition for retrieval-induced forgetting? Psychonomic Bulletin \& Review, 13, 10231027.

Wiley, J. (1998). Expertise as a mental set: The effects of domain knowledge in creative problem solving. Memory \& Cognition, 26, 716-730.

Williams, C. C., \& Zacks, R. T. (2001). Is retrieval induced forgetting an inhibitory process? American Journal of Psychology, 114, 329-354.

Wimber, M., Rutschmann, R. M., Greenlee, M. W., \& Bäuml, K. -H. (2009). Retrieval from episodic memory: Neural mechanisms of interference resolution. Journal of Cognitive Neuroscience, 21, 538-549.

- volume 4, no. 2 (Spring 2012) 